\title{
INVARIANTS OF THE LUSTERNIK-SCHNIRELMANN TYPE AND THE TOPOLOGY OF CRITICAL SETS
}

\author{
MÓNICA CLAPP AND DIETER PUPPE
}

\begin{abstract}
We introduce and study in detail generalizations of the notion of Lusternik-Schnirelmann category which give information about the topology of the critical set of a differentiable function. We also improve a result of $T$. Ganea about the equality of the strong category and the category (even in the classical case).
\end{abstract}

The category $\operatorname{cat}(X)$ of a space $X$ in the sense of Lusternik and Schnirelmann [14] is the smallest number $k$ such that there exists a covering $\left\{X_{1}, \ldots, X_{k}\right\}$ of $X$ (of a certain kind, cf. 1.2(1)) for which each inclusion $X_{j} \subset X$ is nullhomotopic. The motivation for introducing this concept was that it gives a lower bound for the number of critical points of a function. More precisely, if $M$ is a closed differentiable manifold and $f$ is a differentiable real function on $M$ then the number of critical points of $f$ is at least $\operatorname{cat}(M)$.

We propose the following generalization: If $\mathscr{A}$ is any class of spaces we replace the condition that $X_{j} \subset X$ is nullhomotopic by requiring that it factors through some $A \in \mathscr{A}$ up to homotopy and we obtain the notion of $\mathscr{A}$-category, $\mathscr{A}$-cat $(X)$. If $\mathscr{A}$ consists only of the one-point space, this is the classical cat $(X)$. If $\mathscr{A}$ is the class of $q$-connected spaces $\mathscr{A}$-cat is the " $q$-dimensional homotopy category" introduced by Fox in [7]. Another interesting example is the class $\mathscr{A}$ of $q$-dimensional spaces.

If $f: M \rightarrow \mathbf{R}$ is as above then $\mathscr{A}$-cat $(M)$ does not give any new information about the number of critical points, because it is less than or equal to cat $(M)$. It does give, however, under certain conditions, some new information on the topological structure of the critical set. Roughly, one can say that either there are at least $\mathscr{A}$-cat $(M)$ critical values of $f$ or there is one critical value $\gamma$ of $f$ such that the corresponding set of critical points $K \cap f^{-1}(\gamma)$ is not of the homotopy type of any space in $\mathscr{A}$ (cf. $\$ 2$ for more details).

Quite a number of papers have appeared on Lusternik-Schnirelmann category and related notions (cf. [13] for a survey). In particular there has been a revival of interest in recent years. We shall present a systematic theory for our more general

Received by the editors November 25, 1985 .

1980 Mathematics Subject Classification (1985 Revision). Primary 55M30; Secondary 58E05, 55P50.

Key words and phrases. Lusternik-Schnirelmann category, abstract critical point theory, homotopy covering, sectional category, strong category.

The authors wish to thank the Deutscher Akademischer Austauschdienst for financial support. The second author wishes also to thank the Instituto de Matemáticas of the National University of Mexico for its hospitality and support. 
notion which contains most of the classical results as special cases. But generality is not our only purpose. Our proofs are not more complicated than the more special ones in the literature. We think that many of them are even simpler and give better insight. This enabled us in particular to obtain an improvement of a classical result of T. Ganea [9] concerning the notion of strong category.

It might seem more natural to define the category of a space $X$ in terms of subspaces of it which are contractible in themselves and not only in $X$. However, the number $C X$ thus obtained is not an invariant of the homotopy type of $X$ (cf. [7, 40]). Ganea studied the minimum value of $C Y$ for all spaces $Y$ of the same homotopy type as $X$ and called it the strong category $\operatorname{Cat}(X)$ of $X$. It is known that

$$
\operatorname{cat}(X) \leqslant \operatorname{Cat}(X) \leqslant \operatorname{cat}(X)+1
$$

(the first inequality is trivial, for the second cf. [25]) and examples of both possibilities are also known (cf. [1, 17]). Now, Ganea proved [9, Theorem 1.3] that $\operatorname{Cat}(X)$ equals cat $(X)=k$ if $X$ is $p$-connected and

$$
\operatorname{dim} X \leqslant(k+1)(p+1)-3 .
$$

We shall not only generalize this result to $\mathscr{A}$-category for many classes $\mathscr{A}$, but we will also replace the last condition by

$$
\operatorname{dim} X \leqslant(2 k-1)(p+1)-3,
$$

$(5.8,5.9)$, which improves Ganea's result for all $k>2$. The whole theory works in a $G$-equivariant setting, where $G$ is a compact Lie group (partly even for more general groups), and Fadell's notion of $G$-category is also an example of our general notion (cf. 1.2(2)). However, for expository purposes, we shall present here mainly the nonequivariant version and just point out once in a while how the theory goes through to the $G$-equivariant case.

We shall work entirely in the category of compactly generated weakly Hausdorff spaces.

\section{Category with respect to a class of spaces.}

1.1 Let $\mathscr{A}$ be a class of spaces which contains at least one nonempty space. We shall say that a subspace $X^{\prime}$ of a space $X$ is deformable in $X$ to $\mathscr{A}$ if the inclusion $i$ : $X^{\prime} \subset X$ factors through some space in $\mathscr{A}$ up to homotopy, i.e. if there exist $A \in \mathscr{A}$ and maps $\alpha: X^{\prime} \rightarrow A$ and $\beta: A \rightarrow X$ such that $\beta \alpha$ is homotopic to $i$. A finite numerable covering $\left\{X_{1}, \ldots, X_{k}\right\}$ of $X$ such that each $X_{j}$ is deformable in $X$ to $\mathscr{A}$ will be called an $\mathscr{A}$-categorical covering of $X$. We define the $\mathscr{A}$-category $\mathscr{A}$-cat $(X)$ of $X$ to be the smallest cardinality $k$ of such a covering. If no such covering exists let $\mathscr{A}$-cat $(X)=\infty$. More generally: the $\mathscr{A}$-category $\mathscr{A}$-cat $(f)$ of a map $f: X \rightarrow Y$ is the smallest cardinality $k$ of a finite numerable covering $\left\{X_{1}, \ldots, X_{k}\right\}$ of $X$ such that for each $j=1, \ldots, k$ the restriction of $f \mid X_{j}: X_{j} \rightarrow Y$ factors through some space in $\mathscr{A}$ up to homotopy. Such a covering will be called an $\mathscr{A}$-categorical covering associated to $f$. Again if no such covering exists then $\mathscr{A}$-cat $(f):=\infty$.

Observe that $\mathscr{A}$-cat $(X)$ is the $\mathscr{A}$-category of the identity map of $X$. These definitions can be extended in the obvious way to the $G$-equivariant setting where $G$ is a topological group. Then of course the $X_{j}$ 's have to be $G$-invariant subspaces of $X$ and the "numerating functions" have to be also $G$-invariant. 
1.2 EXAMPLES. (1) Let $\mathscr{P}$ be the class which consists only of the one-point space. Then $\mathscr{P}$-cat $(X)$ is nothing but the Lusternik-Schnirelmann category cat $(X)$ of $X$, except that classically the coverings of $X$ considered in the definition were either open or closed instead of numerable (cf. [7]). If $X$ is a normal space our definition is equivalent to the one with open coverings and if $X$ is an ANR then it is also equivalent to the one with closed coverings.

(2) We give now an example in the $G$-equivariant setting: Let $G-\mathscr{P}$ be the class of all homogeneous spaces $G / H$, where $H$ is a closed subgroup of $G$. We shall denote $G$ - $\mathscr{P}_{\text {-cat }}(X)$ simply by $G$-cat $(X)$ and call it the $G$-equivariant category of the $G$-space $X$. This is the most natural extension of the classical notion to the equivariant case (cf. [6, 2.1]).

(3) If $\mathscr{C}_{q}$ is the class of all $q$-connected $\mathrm{CW}$-complexes, $q \geqslant 0$, then $\mathscr{C}_{q}$-cat $(X)$ is the " $q$-dimensional homotopy category of $X$ " introduced by Fox in [7, 15]. Here we shall call it the q-connective category of $X$ and denote it simply by cat ${ }_{q}(X)$.

(4) Let $G-\mathscr{D}^{q}$ be the class of all $G$-CW-complexes of dimension $\leqslant q$. We denote by $G$-cat ${ }^{q}(X)$ the $G-\mathscr{D}^{q}$-category of the $G$-space $X$, or simply by cat ${ }^{q}(X)$ if $G$ is trivial, and call it the $q$-dimensional (equivariant) category of $X$. This notion plays an important role for example in [16].

1.3 Some easy properties of $\mathscr{A}$-category are the following:

(1) $\mathscr{A}$-cat $(f: X \rightarrow Y)=0$ iff $X=\varnothing$.

(2) $\mathscr{A}$-cat $(X)=1$ iff $X$ is dominated by some space in $\mathscr{A}$ and $X \neq \varnothing$.

(3) For any two maps $f: X \rightarrow Y$ and $g: Y \rightarrow Z$,

$$
\mathscr{A} \text {-cat }(g f) \leqslant \min \{\mathscr{A} \text {-cat }(f), \mathscr{A} \text {-cat }(g)\} \text {. }
$$

In particular

$$
\mathscr{A} \text {-cat }(f) \leqslant \min \{\mathscr{A} \text {-cat }(X), \mathscr{A} \text {-cat }(Y)\} .
$$

(4) If $\left\{X_{1}, X_{2}\right\}$ is a numerable covering of $X$ then for any map $f: X \rightarrow Y$

$$
\mathscr{A} \text {-cat }(f) \leqslant \mathscr{A} \text {-cat }\left(f \mid X_{1}\right)+\mathscr{A} \text {-cat }\left(f \mid X_{2}\right) \text {. }
$$

(5) $\mathscr{A}$-cat $(f)$ depends only on the homotopy class of $f$.

A formal consequence of (3) and (5) is the following

1.4 Proposition. If $X$ is dominated by $Y$ then $\mathscr{A}$-cat $(X) \leqslant \mathscr{A}$-cat $(Y)$. In particular $\mathscr{A}$-category is an invariant of the homotopy type of $X$.

Finally observe that if $\mathscr{A} \subset \mathscr{B}$ then $\mathscr{B}$-cat $(f) \leqslant \mathscr{A}$-cat $(f)$ for any map $f$. Applying this to the examples in 1.2 we have that

$$
\operatorname{cat}_{q-1}(f) \leqslant \operatorname{cat}_{q}(f) \leqslant \operatorname{cat}(f) \text { and } \operatorname{cat}^{q+1}(f) \leqslant \operatorname{cat}^{q}(f) \leqslant \operatorname{cat}(f) .
$$

Before studying $\mathscr{A}$-cat in detail we shall establish its relation with the critical sets of differentiable functions.

2. A-category and critical sets. Let $M$ be a paracompact $C^{1}$-Banach manifold (possibly with boundary) and let $f: M \rightarrow \mathbf{R}$ be a $C^{1}$-function. One may also consider the $G$-equivariant case where $G$ is a compact Lie group acting differentiably on $M$ and $f$ is $G$-invariant. What follows holds also in this case (with the suitable obvious modifications). Let $K$ be the critical set of $f$, i.e. the set of all points in $M$ where the derivative of $f$ vanishes. Then $f(K)$ is the set of critical values and 
$\mathbf{R} \backslash f(K)$ is the set of regular values of $f$. For any $\alpha \in \mathbf{R}$ we write

$$
\left.\left.M_{\alpha}:=f^{-1}\right]-\infty, \alpha\right] \text { and } K_{\alpha}:=K \cap f^{-1}(\alpha) .
$$

Our aim is to show, by extending the classical Lusternik-Schnirelmann method [14, 21], how $\mathscr{A}$-category may be used to obtain new information about the topology of the sets $K_{\alpha}$.

2.1 We need some assumptions on $M$ and $f$. If $X, X^{\prime}$ and $X^{\prime \prime}$ are subsets of $M$ we say that $X$ is deformable (in $M$ ) into $X^{\prime}\left(\operatorname{rel} X^{\prime \prime}\right)$ if there exists a homotopy $h_{t}$ : $X \rightarrow M, t \in[0,1]$, such that $h_{0}$ is the inclusion and $h_{1}(X) \subset X^{\prime}$ (and $h_{t}(X)$ is independent of $t$ if $\left.x \in X^{\prime \prime}\right)$. Consider the following deformation conditions:

$\left(\mathrm{D}_{1}\right)$ For any $\alpha$ in the interior of the set of regular values of $f$ there is an $\varepsilon>0$ such that $M_{\alpha+\varepsilon}$ is deformable into $M_{\alpha-\varepsilon}$.

$\left(\mathrm{D}_{2}\right)$ For any isolated critical value $\alpha$ of $f$ and any neighborhood $V$ of $K$ there is an $\varepsilon>0$ such that $M_{\alpha+\varepsilon} \backslash V$ is deformable into $M_{\alpha-\varepsilon}$.

$\left(\mathrm{D}_{3}\right)$ If $\alpha>\sup f(K)$ then $M$ is deformable into $M_{\alpha}$.

It is not hard to see (and perhaps not very important) that $\left(D_{3}\right)$ is a consequence of the following slightly stronger version of $\left(D_{1}\right)$ :

$\left(\mathrm{D}_{1}^{\prime}\right)$ For any $\alpha \in \operatorname{int}(\mathbf{R} \backslash f(K))$ there is an $\varepsilon>0$ such that $M_{\alpha+\varepsilon}$ is deformable into $M_{\alpha-\varepsilon}$ rel $M_{\alpha-1}$.

Following Fadell [6] (but slightly different in detail) we call $\left(\mathrm{D}_{1}\right),\left(\mathrm{D}_{2}\right)$ and $\left(\mathrm{D}_{3}\right)$ together the generalized Palais-Smale condition (GPS).

2.2 What are sufficient conditions for (GPS) to hold? Assume first that $M$ has no boundary. If $M$ is a Hilbert manifold then (being paracompact) it has a Riemannian structure. If in addition $f: M \rightarrow \mathbf{R}$ is a $C^{2}$-function and a proper map (which is only possible if $M$ has finite dimension) then standard methods of integrating the gradient field $\nabla f$ prove $\left(\mathrm{D}_{1}\right),\left(\mathrm{D}_{2}\right),\left(\mathrm{D}_{3}\right)$ and $\left(\mathrm{D}_{1}^{\prime}\right)$, cf. e.g. $[12,6.2]$.

This was extended by Palais and Smale $[19,22,24]$ to the case of a $C^{2}$-function $f$ on a Hilbert manifold $M$ (without boundary) satisfying

(C) For any $S \subset M$ such that $f$ is bounded but $\|\nabla f\|$ is not bounded away from zero there exists a critical point in the closure $\bar{S}$.

In [21, 5.11] Palais extended it further to include all Banach manifolds $M$ of a certain kind (complete $C^{2}$-Finsler manifolds) and all $C^{2-}$-functions ( $C^{1}$-functions whose derivative is locally Lipschitz) $f: M \rightarrow \mathbf{R}$ satisfying a condition obtained from (C) by replacing the gradient $\nabla f$ by a "pseudo-gradient" [21, 4.1 and 5.2].

Finally one may allow $M$ to have a boundary $\partial M$ if there exists a pseudo-gradient field on $M$ which nowhere on $\partial M$ points to the inside of $M$.

Everything in 2.1 and 2.2 has a straightforward extension to the equivariant case for a compact Lie group $G$ (if necessary one integrates over $G$ ).

Now we are ready to state our version of Lusternik and Schnirelmann's main result (cf. [14, II, §4] and [21, 7.1]). We write $\mathscr{A}$-cat ${ }_{M}(X)$ for $\mathscr{A}$-cat $(X \subset M)$.

2.3 THEOREM. Let $M$ be a paracompact $C^{1}$-Banach manifold and $f: M \rightarrow \mathbf{R} a$ $C^{1}$-function satisfying the generalized Palais-Smale condition. Consider the function

$$
m: \mathbf{R} \rightarrow \mathbf{N} \cup\{\infty\}, \quad \alpha \mapsto \mathscr{A}-\text { cat }_{M}\left(M_{\alpha}\right)
$$


where $\mathscr{A}$ is a class of spaces having the homotopy type of a $C W$-complex ( $G$-CW-complex in the equivariant case). Then

(i) The function $m$ is (weakly) increasing.

(ii) In the interior of the set of regular values $m$ is locally constant.

(iii) At any $\alpha \in \mathbf{R}$ which is an isolated critical value of $f$ the function $m$ jumps at most by $\mathscr{A}_{\text {-cat }}{ }_{M}\left(K_{\alpha}\right)$.

(iv) $m(\alpha)=\mathscr{A}$-cat $(M)$ for all $\alpha>\sup f(K)$.

Observe that the hypothesis on $\mathscr{A}$ is satisfied in all our examples 1.2. The formulation of the theorem looks a little different from the traditional one, but by observing that

$$
c_{i}:=\inf \left\{\sup f(X) \mid \mathscr{A} \text {-cat }{ }_{M}(X) \geqslant i\right\}=\inf \{\alpha \mid m(\alpha) \geqslant i\}
$$

(if finite) is just the point where $m$ jumps from some value $\leqslant i-1$ to some value $\geqslant i$, it is easy to translate one into the other. As in the classical case the proof depends just on a few simple properties of the set function

$$
M \supset X \stackrel{n}{\rightarrow} n(X):=\mathscr{A}-\mathrm{cat}_{M}(X)
$$

namely the following.

(1) Monotonicity. $X^{\prime} \subset X \subset M \Rightarrow n\left(X^{\prime}\right) \leqslant n(X)$.

(2) Subadditivity. If $X_{1}, X_{2}$ form a numerable covering of $X \subset M$ then $n(X) \leqslant n\left(X_{1}\right)+n\left(X_{2}\right)$.

(3) Deformation invariance. If $X \subset M$ is deformable (in $M$ ) into $X^{\prime}$ then $n(X) \leqslant n\left(X^{\prime}\right)$.

(4) Continuity. If $X$ is closed in $M$ then there is a neighborhood $U$ of $X$ such that $n(U)=n(X)$.

For $n=\mathscr{A}$-cat ${ }_{M}$ properties (1)-(3) are trivial consequences of the definition. Continuity (4) is easily proved using the fact that $M$ is a $(G)$-ANR and $(G-) C W$ complexes are ( $G$-)ANEs (cf. Appendix B for details).

The rest of the proof of the theorem is obvious: (i) follows directly from (1), (ii) from (3) using $\left(D_{1}\right)$ in 2.1, (iv) also from (3) using $\left(D_{3}\right)$. To prove (iii) take a neighborhood $U$ of $K_{\alpha}$ such that $n(U)=n\left(K_{\alpha}\right)$. Let $V$ be a closed neighborhood of $K_{\alpha}$ in the interior of $U$ and choose $\varepsilon>0$ as i $2.1\left(\mathrm{D}_{2}\right)$. Then

$$
\begin{aligned}
m(\alpha+\varepsilon) & =n\left(M_{\alpha+\varepsilon}\right) \\
& \leqslant n\left(M_{\alpha+\varepsilon} \backslash V\right)+n(U) \quad \text { by }(2) \\
& \leqslant n\left(M_{\alpha-\varepsilon}\right)+n\left(K_{\alpha}\right) \quad \text { by }\left(\mathrm{D}_{2}\right) \text { and }(3) \\
& =m(\alpha-\varepsilon)+\mathscr{A}-\text { cat }_{M}\left(K_{\alpha}\right) . \quad
\end{aligned}
$$

2.4 Corollary. If $f: M \rightarrow \mathbf{R}$ and $\mathscr{A}$ are as in Theorem 2.3 and if in addition $f$ is bounded below then

$$
\mathscr{A} \text {-cat }(M) \leqslant \sum_{\gamma \in \mathbf{R}} \mathscr{A}-\mathrm{cat}_{M}\left(K_{\gamma}\right)
$$


This is trivial if $f$ has infinitely many critical values. Otherwise every $\gamma \in \mathbf{R}$ is either a regular or an isolated critical value of $f$. Choosing $\alpha<\inf f(M)$ and $\beta>\sup f(K)$ we have $m(\alpha)=0$ and

$$
\begin{aligned}
\mathscr{A} \text {-cat }(M) & =m(\beta) \quad \text { by (iv) } \\
& \leqslant \sum_{\gamma \in \mathbf{R}} \mathscr{A} \text {-cat }_{M}\left(K_{\gamma}\right) \quad \text { by (i)-(iii). }
\end{aligned}
$$

This implies in particular that $\mathscr{A}$-cat $(M)$ is a lower bound for the number of critical points of $f$, but since $\mathscr{A}$-cat $(M) \leqslant \operatorname{cat}(M)$ this is already well known (cf. [21, 7.2]). The corresponding assertion in the equivariant setting will be discussed in 2.9 below.

2.5 Another useful consequence is that if $\mathscr{A}$ and $f$ are like in 2.4 then either $f$ has at least $\mathscr{A}$-cat $(M)$ critical values or there is a critical value $\gamma$ of $f$ such that $\mathscr{A}$-cat ${ }_{M}\left(K_{\gamma}\right)>1$. This means that the critical set $K_{\gamma}$ at the level $\gamma$ (and hence the whole critical set) cannot be deformed in $M$ to $\mathscr{A}$. In particular $K_{\gamma}$ is not dominated by any space in $\mathscr{A}$. We shall discuss now what this means in the examples given in 1.2 .

2.6 If $f: M \rightarrow \mathbf{R}$ is as above and if it has less than cat $(M)$ critical values then its critical set $K$ is not contractible in $M$ (cf. 1.2, Example 1). In particular this is true if $M=\mathbf{R} P^{n}, \mathbf{C} P^{n}$ or $\mathbf{H} P^{n}$ and $f: M \rightarrow \mathbf{R}$ is a $C^{2}$-function having at most $n$ critical values (cf. 3.2). We will actually say a lot more about $K$ in 2.8 .

2.7 Let $\mathscr{A}=\mathscr{D}^{q}$ be the class of $q$-dimensional CW-complexes (1.2, Example 4) and let $f: M \rightarrow \mathbf{R}$ be a $C^{1}$-function bounded below which satisfies (GPS). Then either $f$ has at least $\operatorname{cat}^{q}(M)$ critical values or the critical set $K$ of $f$ has covering dimension greater than $q$. This follows from 2.5 because if $\operatorname{dim} K \leqslant q$ then, since $M$ is an ANR [20, p. 3] the inclusion $K \subset M$ factors up to homotopy through some $q$-dimensional simplicial complex, namely the nerve of a suitable covering of $K$.

As a specific example let $M=\mathbf{R} P^{n}, \mathbf{C} P^{n}$ or $\mathbf{H} P^{n}$ and let $f: M \rightarrow \mathbf{R}$ be a $C^{2}$-function with precisely $k$ critical values. Then the dimension of the critical set of $f$ is at least $d \cdot[n / k]$ where $d=1,2$ or 4 resp.

Proof. Let $r=[n / k]$. Then $k \leqslant n / r<$ cat $^{d r-1}(M)$ by 3.2 below.

2.8 Now let $\mathscr{A}=\mathscr{C}_{q}$ be the class of $q$-connected CW-complexes (1.2, Example 3 ) and $f: M \rightarrow \mathbf{R}$ be again a $C^{1}$-function bounded below satisfying (GPS). Let $M$ be $p$-connected $(p \geqslant 0)$ and assume that $f$ has less than cat $_{q}(M)$ critical values (hence $q>p)$. Then there exists an $r, p<r \leqslant q$, such that the Čech cohomology group

$$
\check{H}^{r}\left(K ; \pi_{r} M\right)=\left[K, K\left(\pi_{r} M, r\right)\right]
$$

of the critical set $K$ of $f$ is nonzero. For otherwise one could lift the inclusion $K \subset M$ to the $q$-connective covering space of $M$ contradicting 2.5 .

For projective spaces this gives the following: If $f: M \rightarrow \mathbf{R}$ is a $C^{2}$-function with at most $n$ critical values then

$$
\begin{array}{ll}
\check{H}^{1}(K ; \mathbf{Z} / 2) \neq 0 & \text { if } M=\mathbf{R} P^{n}, \\
\check{H}^{2}(K ; \mathbf{Z}) \neq 0 & \text { if } M=\mathbf{C} P^{n}, \\
\check{H}^{4}(K ; \mathbf{Z}) \neq 0 & \text { if } M=\mathbf{H} P^{n} .
\end{array}
$$


Proof. Apply the preceding result with $p=q-1$ and $q=1,2,4$ respectively and use 3.2(3).

2.9 Let now $G$ be a compact Lie group and $\mathscr{A}=G-\mathscr{P}$ be the class of homogeneous spaces (1.2, Example 2). Let $M$ be a differentiable $G$-Banach manifold and $f$ : $M \rightarrow \mathbf{R}$ be a $G$-invariant $C^{1}$-function bounded below and satisfying (GPS) in the equivariant sense. This is certainly satisfied if $M$ is compact without boundary and $f$ is $C^{2}$, cf. also 2.2. Obviously the critical set $K$ of $f$ is a union of orbits, and it follows from 2.4 that $f$ has at least $G$-cat $(M)$ critical orbits $[6,2.6]$. Note that $G$-cat $(M)$ may be greater than both the ordinary category of $M$ and of its orbit space $M / G$.

But one can do a little better: Call two orbits of $M$ equivalent if they have the same type, $f$ has the same value on them and their inclusions into $M$ are $G$-homotopic. The latter means that we may write the orbits as $G x$ and $G y$, where $x$ and $y$ have the same isotropy group $H$ and lie in the same path component of the fixed point set $M^{H}$. The result is then that either the set of critical orbits is not discrete in $M / G$ (hence infinite) or the number of equivalence classes of orbits in $K$ is at least $G$-cat $(M)$. And the proof follows again from 2.4 because if the set of critical orbits is discrete then the unions of equivalence classes of orbits in $K_{\gamma}$ form a $G$ - $\mathscr{P}$-categorical covering of $K_{\gamma}$ associated to $K_{\gamma} \subset M$.

We now turn to study $\mathscr{A}$-category in detail. Like in the classical case $[13,1.3]$ one obtains lower bounds for $\mathscr{A}$-cat $(X)$ in terms of the multiplicative structure of the cohomology of $X$.

3. $\mathscr{A}$-category and cohomology. Let $T^{*}$ be a $G$-equivariant multiplicative cohomology theory. By this we mean that $T^{*}$ is defined on the category of $G$-spaces and $G$-maps, it satisfies the axioms of exactness, $G$-homotopy and excision (but not necessarily a dimension axiom) and it has a cup-product structure.

If $\mathscr{A}$ is a class of spaces and $Y$ is a space we denote by $T_{\mathscr{\infty}}^{*} Y$ the intersection of the kernels of the homomorphisms $T^{*} Y \rightarrow T^{*} A$ induced by all maps $A \rightarrow Y$ for all $A \in \mathscr{A}$.

For example, let $G$ be trivial and $T^{*}$ be ordinary singular cohomology $H^{*}$. If $\mathscr{A}=\mathscr{D}^{q}$ (cf. 1.2) then $H_{\mathscr{A}}^{n} Y=0$ for $n \leqslant q$ and $H_{\mathscr{Q}}^{n} Y=H^{n} Y$ for $n>q$. For $\mathscr{A}=\mathscr{P}$ the assertion is the same as for $\mathscr{A}=\mathscr{D}^{0}$. If $\mathscr{A}=\mathscr{C}_{q}$ then $H_{\mathscr{A}}^{n} Y=0$ for $n=0$ and $H_{\mathscr{Q}}^{n} Y=H^{n} Y$ for $0<n \leqslant q$.

Let now $f: X \rightarrow Y$ be a map such that $\mathscr{A}$-cat $(f) \leqslant k$ and take $k$ elements $\alpha_{1}, \ldots, \alpha_{k}$ in $T_{\mathscr{A}}^{*} Y$. If $\left\{X_{1}, \ldots, X_{k}\right\}$ is an $\mathscr{A}$-categorical covering associated to $f$ (cf. 1.1) then $f^{*} \alpha_{j}$ goes to zero in $T^{*} X_{j}$. Hence it is in the image of $T^{*}\left(X, X_{j}\right)$ which implies that

$$
f^{*}\left(\alpha_{1} \cdots \alpha_{k}\right)=f^{*} \alpha_{1} \cdots f^{*} \alpha_{k}=0 .
$$

Thus we have proved that

3.1 Proposition. If there are $k-1$ (not necessarily distinct) elements in $T_{\mathscr{A}}^{*} Y$ whose product does not go to zero under $f^{*}: T^{*} Y \rightarrow T^{*} X$ then $\mathscr{A}$-cat $(f) \geqslant k$. 
3.2 Examples. Applying this with $T^{*}=$ singular cohomology with $\mathbf{Z} / 2$-coefficients we obtain the following lower bounds for the classical, the $q$-dimensional and the $q$-connective categories of projective spaces $M=\mathbf{R} P^{n}, \mathbf{C} P^{n}$ or $\mathbf{H} P^{n}$ with $d=1$, 2 or 4 resp.:

$$
\begin{array}{ll}
\operatorname{cat}(M) \geqslant n+1, & \\
\operatorname{cat}^{d q+e}(M) \geqslant[n /(q+1)]+1 & \text { if } 0 \leqslant e<d, \\
\text { cat }_{q}(M) \geqslant n+1 & \text { if } q \geqslant d, \\
\text { cat }_{q}(M)=1 & \text { if } q<d .
\end{array}
$$

All these inequalities are in fact equalities, as we shall prove in 4.7.

Other well-known bounds (both lower and upper) for the classical cat [10] may also be generalized to $\mathscr{A}$-cat, at least for some classes $\mathscr{A}$. For this one needs however some equivalent definitions of $\mathscr{A}$-cat. We shall give those in the next section.

\section{4. $\mathscr{A}$-category and sectional category.}

4.1 Let $X$ be a space and $\mathscr{A}$ be a class of spaces. One can sometimes find a space $U$ in $\mathscr{A}$ and a map $u: U \rightarrow X$ such that, for every $A \in \mathscr{A}$, every map $\beta: A \rightarrow X$ can be lifted to $U$ up to homotopy, i.e. there exists $\tilde{\beta}: A \rightarrow U$ such that $u \tilde{\beta}$ is homotopic to $\beta$. A map $u: U \rightarrow X$ having this property will be called $\mathscr{A}$-universal. (More systematically one could call it weakly terminal in $\mathscr{A}$ over $X$.)

4.2 EXAMples (recall 1.2). (1) If $\mathscr{A}=\mathscr{P}$ is the one-point space class and if $X$ is pathwise connected then any map $u: \mathrm{pt} \rightarrow X$ is $\mathscr{A}$-universal.

(2) In the equivariant case a $G$-space admits a $G$ - $\mathscr{P}$-universal map if and only if there exists $x_{0} \in X$ such that in every orbit one can find a point $x$ which may be joined to $x_{0}$ by a path in the fixed point set of the isotropy group $G_{x}$. Since this is a strong restriction it may be better, if a universal map is needed, to consider $G-\mathscr{D}^{0}$ instead of $G-\mathscr{P}$ (cf. (4) below).

(3) Let $\mathscr{A}=\mathscr{C}_{q}$ be the class of $q$-connected CW-complexes and $X$ be pathwise connected. Take any weak equivalence $\bar{X} \rightarrow X$ from a CW-complex $\bar{X}$ to $X$ and let $\tilde{X}^{q} \rightarrow \bar{X}$ be the $q$-connective covering fibration of $\bar{X}$ (cf. for example [11, 17.16]). Then $\tilde{X}^{q} \in \mathscr{C}_{q}$ and the composition $u: \tilde{X}^{q} \rightarrow \bar{X} \rightarrow X$ induces isomorphisms of the homotopy groups from dimension $q+1$ on. Hence it is $\mathscr{C}_{q}$-universal.

(4) Consider now the case of (equivariant) $q$-dimensional category, i.e. $\mathscr{A}=G-\mathscr{D}^{q}$. For any $G$-space $X$ there is a $G$-CW-complex $\bar{X}$ and a weak $G$-homotopy equivalence $\bar{X} \rightarrow X[26,3.7]$. If $X^{(q)}$ is the $q$-skeleton of $\bar{X}$ then $u: X^{(q)} \subset \bar{X} \rightarrow X$ is $G$ - $\mathscr{D}^{q}$-universal.

Let $u: U \rightarrow X$ be $\mathscr{A}$-universal. Observe that a subspace $X^{\prime}$ of $X$ is deformable in $X$ to $\mathscr{A}$ if and only if the inclusion $X^{\prime} \subset X$ can be lifted to $U$ up to homotopy. This motivates the following definition and proves the next proposition:

4.3 Definition [13, §8]. The sectional category $\sec (\mathrm{g})$ of a map $g: B \rightarrow X$ is the smallest cardinality $k$ of a finite numerable covering $\left\{X_{1}, \ldots, X_{k}\right\}$ of $X$ such that each inclusion $X_{j} \subset X$ can be lifted to $B$ up to homotopy.

4.4 Proposition. If $u: U \rightarrow X$ is $\mathscr{A}$-universal then $\mathscr{A}$-cat $(X)=\operatorname{secat}(u)$. 
More generally: Let $f: X \rightarrow Y$ be a map, $u$ : $U \rightarrow Y$ be $\mathscr{A}$-universal and $u^{\prime}$ : $U^{\prime} \rightarrow X$ be the homotopy pullback of $u$ over $f$, i.e. $u^{\prime}$ is the pullback over $f$ of the (Hurewicz-) fibration associated to $u$. Then it is easy to see that $\mathscr{A}$-cat $(f)=\operatorname{secat}\left(u^{\prime}\right)$. We shall now give some characterizations of secat (and hence of $\mathscr{A}$-cat in many interesting cases).

4.5 For any map $g: B \rightarrow X$ we define the $k$-fold join $g_{k}: J_{X}^{k} B \rightarrow X$ of $B$ over $X$ inductively as follows: $g_{1}: J_{X}^{1} B \rightarrow X$ is the (Hurewicz-) fibration associated to $g$. Suppose that $g_{k-1}: J_{X}^{k-1} B \rightarrow X, k>1$, has been defined. Consider the pullback diagram

$$
\begin{array}{cll}
P_{X}^{k-1} B:=J_{X}^{1} B \underset{X}{\times} J_{X}^{k-1} B & \stackrel{g_{1}^{\prime}}{\rightarrow} & J_{X}^{k-1} B \\
g_{k-1}^{\prime} \downarrow & & \downarrow g_{k-1} \\
J_{X}^{1} B & \stackrel{g_{1}}{\rightarrow} & X
\end{array}
$$

and define $J_{X}^{k} B$ to be the double mapping cylinder of $g_{1}^{\prime}$ and $g_{k-1}^{\prime}$ and $g_{k}$ : $J_{X}^{k} B \rightarrow X$ to be the map induced by $g_{k-1}$ and $g_{1}$. Then each $g_{k}$ is a fibration (cf. A.3). We have

4.6 Proposition. secat $(g) \leqslant k$ if and only if $g_{k}: J_{X}^{k} B \rightarrow X$ has a section. In particular if $u: U \rightarrow X$ is $\mathscr{A}$-universal then $\mathscr{A}$-cat $(X) \leqslant k$ if and only if $u_{k}: J_{X}^{k} U \rightarrow X$ has a section.

Proof. Observe that $X^{\prime} \subset X$ can be lifted to $B$ up to homotopy iff $g_{1}: J_{X}^{1} B \rightarrow X$ has a section over $X^{\prime}$. Now, the points of $J_{X}^{k} B$ may be thought of as being represented by $\left(t_{1} b_{1}, \ldots, t_{k} b_{k}\right)$ where all the $b_{j}$ 's lie in the same fiber of $g_{1}$ and the $t_{j}$ 's are nonnegative real numbers such that $t_{1}+\cdots+t_{k}=1$. The proof is now standard. It uses of course the partition of unity (cf. [13, 8.1]).

This generalizes Ganea's characterization of category $[9,2.2]$. As a consequence of 4.6 we obtain an upper bound for secat:

4.7 Corollary. If $g: B \rightarrow X$ is a p-equivalence and $X$ is a $C W$-complex of dimension less than $k(p+1)$ then $\sec a t(g) \leqslant k$.

Proof. This follows from the fact that $g_{k}: J_{X}^{k} B \rightarrow X$ is a $(k(p+1)-1)$ equivalence (cf. A.2).

Hence the inequalities given in 3.2 for the classical, the $q$-dimensional and the $q$-connective categories of projective spaces are in fact inequalities.

We give now another characterization of secat which generalizes $\mathrm{G}$. W. Whitehead's definition of category [27] (see also [13, §5]). Given $g: B \rightarrow X$ we define the $k$-fold wedge $W_{k}^{B} X$ of $X$ under $B$ as follows: Think of $B$ as being embedded into the top of the mapping cylinder $Z_{g}$ of $g$ (in other words: replace $g$ by its associated cofibration). Then $W_{k}^{B} X$ is the subspace of the $k$-fold product $Z_{g}^{k}$ of $Z_{g}$ consisting of all points with at least one coordinate in $B$. For expository purposes let us identify $Z_{g}$ 
with $X$ (i.e. we assume that $g: B \rightarrow X$ is already a closed cofibration). Let then $i_{k}$ : $W_{k}^{B} \subset X^{k}$ be the inclusion and $\Delta: X \rightarrow X^{k}$ be the diagonal map. Then

4.8 Proposition. There is a homotopy pullback diagram

$$
\begin{array}{ccc}
J_{X}^{k} B & \rightarrow & W_{k}^{B} X \\
g_{k} \downarrow & & \downarrow i_{k} \\
X & \stackrel{\Delta}{\rightarrow} & X^{k}
\end{array}
$$

Proof (by induction on $k$ ). For $k=1$ this is obvious. Consider the diagram of inclusions

$$
\begin{array}{ccccc}
W_{k}^{B} X \times X & \leftarrow & W_{k}^{B} X \times B & \rightarrow & X^{k} \times B \\
& \searrow & \downarrow & \swarrow & \\
& & X^{k} \times X & &
\end{array}
$$

Since $B \subset X$ is a closed cofibration the double mapping cylinder of the row is just its pushout $W_{k+1}^{B} X$. Replacing the vertical arrows by fibrations and taking their restrictions over $X$ we obtain

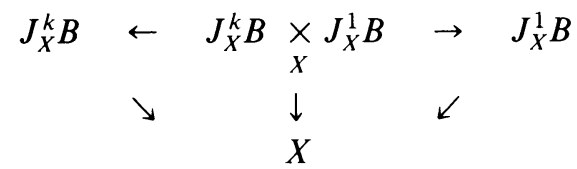

The rest follows from the easy observation that the pullback of a double mapping cylinder is the double mapping cylinder of the pullbacks.

An immediate consequence of 4.8 is (cf. [27, 13; 4.1])

4.9 COROLLARY. secat $(g) \leqslant k$ if and only if the diagonal of $X^{k}$ can be deformed in $X^{k}$ into $W_{k}^{B} X$.

Given maps $h: D \rightarrow B$ and $g: B \rightarrow X$ then obviously secat $(g h) \geqslant \operatorname{secat}(g)$.

A condition for the equality is given by

4.10 Proposition. If $h: D \rightarrow B$ is a q-equivalence and $g: B \rightarrow X$ is a p-equivalence, $p \leqslant q$, and if $X$ is a $C W$-complex of dimension less than or equal to $q+$ $(\operatorname{secat}(g)-1)(p+1)$ then $\operatorname{secat}(g h)=\operatorname{secat}(g)$.

Proof. Let $k=\operatorname{secat}(g)$. We shall prove by induction on $k$ that the map $J_{X}^{k} D \rightarrow J_{X}^{k} B$ induced by $h$ is a $(q+(k-1)(p+1))$-equivalence. Let $k>1$ and consider the diagram

$$
\begin{array}{ccccc}
J_{X}^{1} D & \leftarrow & J_{X}^{1} D \underset{X}{\times} J_{X}^{k-1} D & \rightarrow & J_{X}^{k-1} D \\
\downarrow q & & & \| \\
J_{X}^{1} B & \stackrel{(k-1)(p+1)-1}{\leftarrow} & J_{X}^{1} B \underset{X}{\times} J_{X}^{k-1} D & & J_{X}^{k-1} D \\
\| & & \downarrow q+(k-2)(p+1) & & \downarrow \\
J_{X}^{1} B & \stackrel{(k-1)(p+1)-1}{\leftarrow} & J_{X}^{1} B \underset{X}{\times} J_{X}^{k-1} B & \stackrel{p}{\rightarrow} & J_{X}^{k-1} B
\end{array}
$$


where all arrows are the obvious ones and the numbers near them indicate how high an equivalence they are (recall that $g_{k-1}: J^{k-1} B \rightarrow X$ is a $((k-1)(p+1)-1)$ equivalence, A.2). Now apply Serre's Theorem A.1 twice.

In particular, if $X$ is p-connected, $q \geqslant p$ and $\operatorname{dim} X \leqslant q+\left(\operatorname{cat}_{q}(X)-1\right)(p+1)$ then

$$
\operatorname{cat}_{q}(X)=\operatorname{cat}_{q+1}(X)=\cdots=\operatorname{cat}(X) .
$$

Proof. Apply 4.10 to $u: \tilde{X}^{q+1} \rightarrow \tilde{X}^{q} \stackrel{u}{\rightarrow} X$ and pt $\rightarrow \tilde{X}^{q} \rightarrow X$, with $u: \tilde{X}^{r} \rightarrow X$ as in 4.2(3).

That the condition given in 4.10 is the best possible is shown by the following example: Let

$$
X=\mathbf{S}^{q+1} \times\left(\mathbf{R} P^{(k-1)(p+1)} / \mathbf{R} P^{p}\right) .
$$

Using 3.1 and 4.7 it is easy to see that $\operatorname{cat}_{q}(X)=k$ and cat ${ }_{q+1}(X) \geqslant k+1$ for $q>p$ and $k>1$. (In fact cat ${ }_{q+1}(X)=k+1$, cf. [3].)

5. Strong $\mathscr{A}$-category. The notion of strong category was introduced by T. Ganea in [9]. We start this section by generalizing this notion to our context and by giving some characterizations of it.

5.1 Let $\mathscr{A}$ be a given class of topological spaces. A $k$-fold mapping cylinder diagram with vertex-spaces in $\mathscr{A}$ is a commutative diagram of spaces $X_{\sigma}, \sigma \subset \underline{k}=$ $\{1, \ldots, k\}, \sigma \neq \varnothing$, and maps $f_{\sigma \tau}: X_{\tau} \rightarrow X_{\sigma}, \sigma \subset \tau, \sigma \neq \tau$, such that $X_{j}:=X_{\{j\}} \in \mathscr{A}$ for all $j=1, \ldots, k$. (Schematically such a diagram looks like the 1-skeleton of the barycentric subdivision of the standard $(k-1)$-dimensional simplex, all arrows pointing outward). Let $\Delta_{\sigma}$ denote the geometric realization of the $(\# \sigma-1)$ dimensional simplex generated by $\sigma$ and let $\varepsilon_{\tau \sigma}$ be the face map induced by $\sigma \subset \tau$.

A $k$-fold mapping cylinder with vertex-spaces in $\mathscr{A}$ is the quotient of the disjoint union $\sqcup_{\sigma \subset k}\left(X_{\sigma} \times \Delta_{\sigma}\right)$ obtained by identifying $\left(x, \varepsilon_{\tau \sigma}(t)\right)$ with $\left(f_{\sigma \tau}(x), t\right)$ for all $x \in X_{\tau}, t \in \Delta_{\sigma}, \sigma \subset \tau$.

ExAMPLES. (1) For every map $g: B \rightarrow X$, the $k$-fold join $J_{X}^{k} B$ of $B$ over $X$ is a $k$-fold mapping cylinder with vertex-spaces all equal to $J_{X}^{1} B$.

(2) A covering $\mathscr{U}=\left\{X_{1}, \ldots, X_{k}\right\}$ of $X$ consisting of spaces in $\mathscr{A}$ gives rise to a $k$-fold mapping cylinder diagram with vertex-spaces in $\mathscr{A}$ by taking $X_{\sigma}:=\bigcap_{j \in \sigma} X_{j}$ and $f_{\sigma \tau}: X_{\tau} \subset X_{\sigma}$ to be the inclusion. The $k$-fold mapping cylinder of this diagram is the classifying space $B \mathscr{U}$ of the covering $\mathscr{U}$ and the canonical projection $\pi_{\mathscr{U}}: B \mathscr{U} \rightarrow X$ is a homotopy equivalence if for example $\mathscr{U}$ is numerable [4].

Let $h \mathscr{A}$ denote the class of all spaces having the homotopy type of some space in $\mathscr{A}$.

5.2 Proposition and Definition. The following are equivalent:

(a) $X$ has the homotopy type of a space $X^{\prime}$ which has a numerable covering $\left\{X_{1}^{\prime}, \ldots, X_{k}^{\prime}\right\} \subset h \mathscr{A}$.

(b) $X$ has the homotopy type of a space $X^{\prime}$ which has a covering $\mathscr{U}=\left\{X_{1}^{\prime}, \ldots, X_{k}^{\prime}\right\}$ $\subset$ h⿻्A such that $\pi_{\mathscr{U}}: B \mathscr{U} \rightarrow X^{\prime}$ is a homotopy equivalence.

(c) $X$ has the homotopy type of a $k$-fold mapping cylinder with vertex-spaces in $\mathscr{A}$.

The smallest number $k$ such that one (and hence all) of these assertions is true will be called the strong $\mathscr{A}$-category of $X$. We denote it by $\mathscr{A}$-Cat $(X)$. It is by definition an invariant of the homotopy type of $X$. 
Proof. (a) $\Rightarrow$ (b) and (b) $\Rightarrow$ (c) follow from the remarks made in Example 5.1(2). Now, let $Z$ be a $k$-fold mapping cylinder with vertex spaces in $\mathscr{A}$. The inverse images under the canonical map $Z \rightarrow \Delta_{\underline{k}}$ of the open stars of the vertices of $\Delta_{\underline{k}}$ form a numerable covering of $Z$ whose elements are homotopy equivalent to the vertexspaces of $Z$. This proves (c) $\Rightarrow$ (a).

5.3 ExAmples. (1) If $\mathscr{P}$ is the one-point space class then $h \mathscr{P}$ is the class of contractible spaces and $\mathscr{P}$-Cat $(X)$ is just Ganea's strong category $\operatorname{Cat}(X)[9,1.2]$, except that all spaces involved in his definition are CW-complexes. But in fact if $X$ and all spaces in $\mathscr{A}$ have the homotopy type of $\mathrm{CW}$-complexes then $\mathscr{A}$-Cat $(X)$ is the smallest $k$ such that $X$ has the homotopy type of a CW-complex $K$ which can be covered by $k$ subcomplexes in $h \mathscr{A}$. And the same is true if one replaces the words "a CW-complex $K$ " by "a semisimplicial complex $K$ " or by "a simplicial complex $K$ ", mainly because in all of these cases $\pi_{\mathscr{U}}: B \mathscr{U} \rightarrow K$ is a homotopy equivalence [4, Remark 2].

(2) Other examples are those corresponding to 1.2(2), (3) and (4). We use the notation $G$-Cat, $\mathrm{Cat}_{q}$ and $\mathrm{Cat}^{q}$ for them.

As in $[9,2.1]$ one has also an inductive characterization of $\mathscr{A}$-Cat, namely

5.4 Proposition. $\mathscr{A}$-Cat $(X)=1$ if and only if $X$ has the homotopy type of some space in $\mathscr{A}$ (and $X \neq \varnothing$ ). If $k, n \geqslant 1$, then $\mathscr{A}$-Cat $(X) \leqslant k+n$ if and only if $X$ is homotopy equivalent to the double mapping cylinder of a diagram $X_{1} \leftarrow X_{0} \rightarrow X_{2}$ with $\mathscr{A}$-Cat $\left(X_{1}\right) \leqslant k$ and $\mathscr{A}$-Cat $\left(X_{2}\right) \leqslant n$.

Proof. If $X \simeq X^{\prime}$ and $\left\{X_{1}^{\prime}, \ldots, X_{k+n}^{\prime}\right\}$ is a numerable covering of $X^{\prime}$ let $X_{1}=X_{1}^{\prime} \cup \cdots \cup X_{k}^{\prime}$ and $X_{2}=X_{k+1}^{\prime} \cup \cdots \cup X_{k+n}^{\prime} .\left\{X_{1}, X_{2}\right\}$ is again a numerable covering of $X^{\prime}$, hence $X^{\prime}$ is homotopy equivalent to the double mapping cylinder of $X_{1} \supset X_{1} \cap X_{2} \subset X_{2}$. The converse is also easy.

Observe that, since $\mathscr{A}$-cat is a homotopy invariant, $\mathscr{A}$-cat $(X) \leqslant \mathscr{A}$-Cat $(X)$ for all spaces $X$. Moreover:

5.5 Proposition. $\mathscr{A}$-cat $(X) \leqslant k$ if and only if $X$ is dominated by a space $Z$ with $\mathscr{A}-\operatorname{Cat}(Z) \leqslant k .^{1}$

Proof. If $X$ is dominated by $Z$ then by $1.4 \mathscr{A}$-cat $(X) \leqslant \mathscr{A}$-cat $(Z) \leqslant \mathscr{A}$-Cat $(Z)$. Conversely, let $\left\{X_{1}, \ldots, X_{k}\right\}$ be a numerable covering of $X$ and $\alpha_{i}: X_{i} \rightarrow A_{i}$ and $\beta_{i}$ : $A_{i} \rightarrow X$ be such that $\beta_{i} \alpha_{i} \simeq\left(X_{i} \subset X\right)$, where $A_{i} \in h \mathscr{A}$ and $\beta_{i}$ is a fibration. Let $Z$ be the fiberwise join of $A_{1}, \ldots, A_{k}$ over $X$ (defined like $J_{X}^{k} U$ in 4.5). Then $Z$ is a $k$-fold mapping cylinder with vertex-spaces in $h \mathscr{A}$ and as in $4.6 X$ is a retract of $Z$.

Hence $\mathscr{A}$-cat $(X) \leqslant k$ if and only if $X$ is dominated by a $k$-fold mapping cylinder with vertex-spaces in $\mathscr{A}$ (compare 4.6).

\footnotetext{
${ }^{1}$ Having completed the manuscript we learned about the thesis of Michael J. Hopkins: Some problems in topology (Oxford, 1984). It contains results on "homotopy covers" which are closely related to this proposition.
} 
As in the classical case $\mathscr{A}$-cat and $\mathscr{A}$-Cat differ for many classes $\mathscr{A}$ by at most one (cf. $[25,5])$ :

5.6 Proposition. Suppose that for every finite family of maps $\beta_{j}: A_{j} \rightarrow X$ with $A_{j} \in \mathscr{A}$ there exist a map $\beta: A \rightarrow X$ with $A \in \mathscr{A}$ and maps $\varphi_{j}: A_{j} \rightarrow A$ such that, for each $j, \beta \varphi_{j}$ is homotopic to $\beta_{j}$. Then $\mathscr{A}-\operatorname{Cat}(X) \leqslant \mathscr{A}$-cat $(X)+1$.

Proof. Let $\left\{X_{1}, \ldots, X_{k}\right\}$ be an $\mathscr{A}$-categorical covering of $X$. Then by our assumption there are maps $\alpha_{j}: X_{j} \rightarrow A$ and $\beta: A \rightarrow X$ such that $\beta \alpha_{j}$ is homotopic to $i_{j}: X_{j} \subset X$ for all $j$. Let $Y$ be the double mapping cylinder of

$$
A \sqcup \cdots \sqcup A \stackrel{\alpha_{1} \sqcup \cdots \sqcup \boldsymbol{\alpha}_{k}}{\leftarrow} X_{1} \sqcup \cdots \sqcup X_{k} \stackrel{\left\langle i_{1}, \ldots, i_{k}\right\rangle}{\rightarrow} X .
$$

Since the mapping cylinders of the $\alpha_{j}$ 's form a numerable covering of $Y, \mathscr{A}-\mathrm{Cat}(Y)$ $\leqslant k$. Now let $Y^{\prime}$ be the double mapping cylinder of

$$
A \sqcup \cdots \sqcup A^{\alpha_{1} \sqcup \cdots \sqcup \alpha_{k}} X_{1} \sqcup \cdots \sqcup X_{k} \stackrel{\left\langle\alpha_{1}, \ldots, \alpha_{k}\right\rangle}{\rightarrow} A .
$$

Since $\beta \alpha_{j} \simeq i_{j}, Y$ is homotopy equivalent to the double mapping cylinder of $Y^{\prime} \supset$ $A \stackrel{\beta}{\rightarrow} X$ and since $A$ is a retract of $Y^{\prime}, X$ has the homotopy type of the double mapping cylinder of $A \stackrel{r}{\leftarrow} Y^{\prime} \rightarrow Y$ where $r$ is a retraction. Hence by $5.4 \mathscr{A}$-Cat $(X)$ $\leqslant k+1$.

The hypothesis of 5.6 is certainly satisfied if $\mathscr{A}$ is closed under finite disjoint unions or if there is a $\mathscr{A}$-universal map into $X$.

What we have said so far about strong $\mathscr{A}$-category is true also in the equivariant case without any changes. Our aim is now to give some conditions for the equality $\mathscr{A}$-Cat $(X)=\mathscr{A}$-cat $(X)$ and for this we will assume that all our spaces are (ordinary) $\mathrm{CW}$-complexes. The methods we are going to use do not work in the equivariant case, at least not immediately. We start by proving the following

5.7 Lemma. Let $m>p \geqslant 1$. Suppose that

(a) there is a p-equivalence $\alpha: A \rightarrow X$ with $A$ simply connected and $A^{(m-1)} \in h \mathscr{A}$,

(b) there is an m-equivalence $g: Y \rightarrow J_{X}^{k} A, k \geqslant 2$, and

(c) $\operatorname{dim} Y \leqslant m-1+(k-1)(p+1)$.

Then $\mathscr{A}$-Cat $(Y) \leqslant k$.

Proof (By INDUCTION ON $k$ ). Let $k \geqslant 2$ and consider the diagram

$\begin{array}{cccccc}A^{(m-1)} & \leftarrow & W_{k-1}^{\prime \prime} & \rightarrow & Z_{k-1} & Z \\ \| & {[6]} & \downarrow & {[5]} & \left(n^{\prime}\right) \downarrow \beta_{k-1} & (n) \downarrow \beta \\ A^{(m-1)} & \leftarrow & W_{k-1}^{\prime} & \stackrel{(p)}{\rightarrow} & Y_{k-1} & Z^{\prime} \\ \alpha^{\prime} \downarrow(m-1) & \stackrel{[3]}{\leftarrow} & \downarrow(m-1) & \stackrel{[4]}{ } & \| & (n) \downarrow \alpha \\ Y^{\prime} & ((k-1)(p+1)-1) & W_{k-1} & \stackrel{(p)}{\rightarrow} & Y_{k-1} & Y \\ \downarrow(m) & \stackrel{\leftarrow}{\leftarrow} & \downarrow(m) & \stackrel{[1]}{(2]} & (m) \downarrow & (m) \downarrow g \\ J_{X}^{1} A & ((k-1)(p+1)-1) & P_{X}^{k-1} A & \stackrel{(p)}{\rightarrow} & J_{X}^{k-1} A & J_{X}^{k} A\end{array}$


which is built up starting from the bottom row (cf. 4.6) as follows [1] and [2] are obtained by taking homotopy pullbacks of $g: Y \rightarrow J_{X}^{k} A$. Hence $Y$ is the double mapping cylinder of the second row (from bottom to top). $\alpha^{\prime}: A^{(m-1)} \rightarrow Y^{\prime}$ is a map whose composition with the map $Y^{\prime} \rightarrow J_{X}^{1} A$ below it is homotopic to the inclusion $A^{(m-1)} \subset A \cong J_{X}^{1} A$. [3] is a homotopy pullback and [4] is commutative. $Z_{1}=A^{(m-1)}$ and $\beta_{1}$ is like $\alpha^{\prime}$, and for $k \geqslant 3, Z_{k-1}$ is the $\left(n^{\prime}=m-1+(k-2)\right.$. $(p+1))$-skeleton of $Y_{k-1}$ and $\beta_{k-1}$ is the inclusion. Finally [5] is a homotopy pullback and [6] is commutative. The numbers in parentheses near the arrows indicate how high an equivalence they are. Let now $Z^{\prime}$ and $Z$ be the double mapping cylinders of the third and the fourth rows respectively. By Serre's Theorem A.1 the induced maps $\alpha$ and $\beta$ are $(n=m-1+(k-1)(p+1))$-equivalences. Now, $\mathscr{A}$-Cat $\left(Z_{1}\right)=1$ and for $k \geqslant 3$, since $n^{\prime} \geqslant m$, by induction hypothesis $\mathscr{A}$ $\operatorname{Cat}\left(Z_{k-1}\right) \leqslant k-1$.

Consider now the Mayer-Vietoris sequence of the fourth row. Since $n^{\prime}=$ $\operatorname{dim} Z_{k-1} \leqslant n-2$ (because $p \geqslant 1$ ), the connecting homomorphism $d_{*}: H_{n} Z \rightarrow$ $H_{n-1} W_{k-1}^{\prime \prime}$ is an isomorphism, and since $\operatorname{dim} Y \leqslant n$ the composition $\alpha_{*} \beta_{*} d_{*}^{-1}$ : $H_{n-1} W_{k-1}^{\prime \prime} \rightarrow H_{n} Y$ is an epimorphism onto a free abelian group. So by $[2,2.1]$ there is an $(n-1)$-dimensional CW-complex $K$ and a map $w: K \rightarrow W_{k-1}^{\prime \prime}$ such that $w_{*}$ : $H_{i} K \rightarrow H_{i} W_{k-1}^{\prime \prime}$ is an isomorphism for $i \leqslant n-2$ and the composition $\alpha_{*} \beta_{*} d_{*}^{-1} w_{*}$ : $H_{n-1} K \rightarrow H_{n} Y$ is an isomorphism. Let

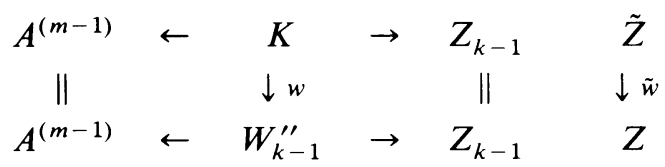

be a commutative diagram (whose bottom row is the top row of the diagram above), let $\tilde{Z}$ be the double mapping cylinder of its top row and $\tilde{w}: \tilde{Z} \rightarrow Z$ be the induced map. Then $\operatorname{dim} \tilde{Z} \leqslant n, \tilde{w}: H_{i} \tilde{Z} \rightarrow H_{i} Z$ is an isomorphism for $i \leqslant n-1$ and, since the connecting homomorphism $d_{*}: H_{n} \tilde{Z} \rightarrow H_{n-1} K$ is again an isomorphism, so is $(\alpha \beta \tilde{w})_{*}: H_{n} \tilde{Z} \rightarrow H_{n} Y$. Hence $\alpha \beta \tilde{w}: \tilde{Z} \rightarrow Y$ induces an isomorphism in homology. Since $A$ is 1-connected and $m>p \geqslant 1, \tilde{Z}$ and $Y$ are also 1-connected. Hence $\alpha \beta \tilde{w}$ : $\tilde{Z} \rightarrow Y$ is in fact a homotopy equivalence. But by $5.4 \mathscr{A}$-Cat $(\tilde{Z}) \leqslant k$.

We are now ready to prove the following

5.8 THEOREM. Suppose that

(a) $\mathscr{A}$-cat $(X)=k, k \geqslant 2$,

(b) there is an A-universal map $u: U \rightarrow X$ which is a p-equivalence, $p \geqslant 1$, with $U$ 1-connected and $U^{(k(p+1)-3)} \in h \mathscr{A}$, and

(c) $\operatorname{dim} X \leqslant(2 k-1)(p+1)-3$.

Then $\mathscr{A}$-Cat $(X)=k$.

Proof. Let $g: X \rightarrow J_{X}^{k} U$ be a section of $u_{k}: J_{X}^{k} U \rightarrow X$ (cf. 4.6). Then $g$ is an $(m=k(p+1)-2)$-equivalence. Now use 5.7 with $A=U$ and $Y=X$.

Applying this to our examples in 1.2 we get

5.9 Corollary. (1) If $X$ is p-connected, $p \geqslant 1$, and $\operatorname{dim} X \leqslant(2 \operatorname{cat}(X)-1)(p+1)-3$

then $\operatorname{Cat}(X)=\operatorname{cat}(X)$. 
(2) If $X$ is p-connected, $p \geqslant 1$, and

$$
\operatorname{dim} X \leqslant\left(2 \operatorname{cat}_{q}(X)-1\right)(p+1)-3
$$

then $\mathrm{Cat}_{q}(X)=\operatorname{cat}_{q}(X)$.

(3) If $X$ is 1 -connected and $\operatorname{dim} X \leqslant\left(2 \operatorname{cat}^{q}(X)-1\right)(q+1)-3$ then $\operatorname{Cat}^{q}(X)=$ $\operatorname{cat}^{q}(X)$.

5.10 RemarKs. Ganea proved Corollary 5.9(1) under the stronger assumption that $\operatorname{dim} X \leqslant(\operatorname{cat}(X)+1)(p+1)-3[9,1.3]$. For $\operatorname{cat}(X)=2$ this condition coincides with ours and it is in fact the best possible (cf. [1 and 17; 2.4]). Now, our same condition appears in a similar result obtained by W. Singhof $[23,6.1]$ and $L$. Montejano [16, 1]. They each used different methods (quite different from ours) to obtain their results. This leads us to the conjecture that this condition is always the best possible.

It is, however, not easy to find counterexamples (the only examples known for $\operatorname{Cat}(X) \neq \operatorname{cat}(X)$ are those of Berstein and Hilton [1] where $\operatorname{cat}(X)=2$ ), mainly because of the fact that $\operatorname{Cat}(X)$ is difficult to compute. It is thus desirable to have better descriptions of $\operatorname{Cat}(X)$. Some nice work in this direction has been done recently by $\mathrm{L}$. Montejano $[\mathbf{1 7}, 2.1]$.

Appendix A: Serre's theorem. The following result played an important role in the proofs of 4.10 and 5.7 .

A.1 Serre's Theorem. Let

$$
\begin{array}{cccccc}
Y & \leftarrow & X_{0} & \stackrel{\varphi}{\rightarrow} & X_{1} & Z_{1} \\
\| & & \psi \downarrow & (\mathrm{D}) & \downarrow g & \downarrow \bar{g} \\
Y & \leftarrow & X_{2} & \stackrel{f}{\rightarrow} & X & Z_{2}
\end{array}
$$

be a homotopy commutative diagram where the right square (D) is a homotopy pullback. Let $Z_{1}$ and $Z_{2}$ be the double mapping cylinders of the rows and $\bar{g}: Z_{1} \rightarrow Z_{2}$ be the map induced by the vertical arrows. Then, if $g$ is an m-equivalence and $f$ is an $n$-equivalence, $\bar{g}$ is an $(m+n+1)$-equivalence.

This theorem can be proved by elementary methods. It is an easy consequence of Mather's generalization $[15,47]$ of Ganea's theorem about the homotopy fiber of the mapping cone of the inclusion of the fiber into the total space of a fibration [8, 1.1]. In order to emphasize the simplicity of the methods and for the reader's convenience we include here also a proof of Mather's result.

A.2 Proposition. Let (D) be a homotopy pullback diagram as in A.1, $Z$ be the double mapping cylinder of $\varphi$ and $\psi$, and $z: Z \rightarrow X$ the map induced by $f$ and $g$. Then $f$,

(i) the homotopy fiber of $z: Z \rightarrow X$ is the join $F_{g} * F_{f}$ of the homotopy fibers of $g$ and

(ii) if $g$ is an m-equivalence and $f$ is an n-equivalence then $z: Z \rightarrow X$ is an $(m+n+1)$-equivalence. 
Proof OF A.1. Consider the diagram

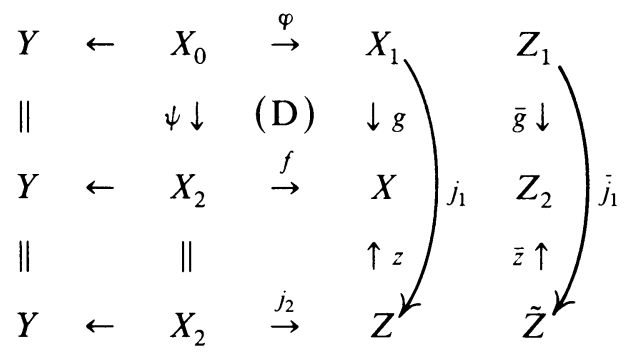

where $j_{1}$ and $j_{2}$ are the inclusions and $Z_{1}, Z_{2}, \tilde{Z}$ are the double mapping cylinders of the rows. The induced map $\bar{j}_{1}: Z_{1} \rightarrow \tilde{Z}$ is a homotopy equivalence. By A.2(ii) $z$ is an $(m+n+1)$-equivalence, so $\bar{z}: \tilde{Z} \rightarrow Z_{2}$ and hence also $\bar{g}: Z_{1} \rightarrow Z_{2}$ are $(m+n$ $+1)$-equivalences.

Proof OF A.2. We may assume that $f$ and $g$ are (Hurewicz-) fibrations and that (D) is an ordinary pullback diagram. Then (i) follows from A.3 below and (ii) follows from the fact that the join of an $(n-1)$-connected space with an $(m-1)$ connected space is $(m+n)$-connected.

A.3 Lemma. Let

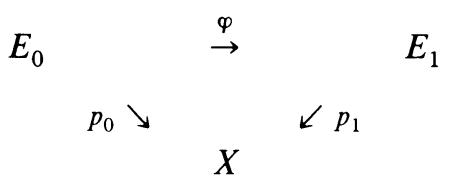

be a map of fibrations. Then any two lifting maps

$$
\Gamma_{0}: E_{0} \times_{X} X^{I} \rightarrow E_{0}^{I} \text { and } \Gamma_{1}: E_{1} \times_{X} X^{I} \rightarrow E_{1}^{I}
$$

for the fibrations $p_{0}$ and $p_{1}$ can be extended to a lifting map $\Gamma$ for the projection $p$ : $Z_{\varphi} \rightarrow X$ (induced by $p_{0}$ and $p_{1}$ ) of the mapping cylinder $Z_{\varphi}$ of $\varphi: E_{0} \rightarrow E_{1}$ onto $X$.

Proof. We define $\Gamma: Z_{\varphi} \times{ }_{X} X^{I} \rightarrow Z_{\varphi}^{I}$ as follows: for $(e, t, \sigma) \in\left(E_{0} \times I\right) \times{ }_{X} X^{I}$, $s \in I$, let

$$
\Gamma(e, t, \sigma)(s)=\left\{\begin{array}{l}
\left(\Gamma_{0}(e, \sigma)(s),\left(t-\frac{1}{2}\right)(1+s)+\frac{1}{2}(1-s)\right) \quad \text { if } \frac{1}{2} \leqslant t \leqslant 1 \\
\left(\Gamma_{0}(e, \sigma)(s), t-\frac{1}{2} s\right) \quad \text { if } 0 \leqslant t \leqslant \frac{1}{2} \text { and } s \leqslant 2 t \\
\Gamma_{1}\left(\varphi\left(\Gamma_{0}(e, \sigma)(2 t)\right), \sigma_{s t}\right)(s-2 t) \quad \text { if } 0 \leqslant t \leqslant \frac{1}{2} \text { and } s \geqslant 2 t
\end{array}\right.
$$

where $\sigma_{2 t}(r)=\sigma(\min \{2 t+r, 1\})$, and for $(e, \sigma) \in E_{1} \times_{X} X^{I}$ let $\Gamma(e, \sigma)=\Gamma_{1}(e, \sigma)$.

Appendix B: Continuity of $\mathscr{A}$-cat ${ }_{M}$. Now we give the proof of 2.3(4), i.e. of the continuity property of the set function $\mathscr{A}$-cat ${ }_{M}$, and for completeness we do it in the equivariant case. Thus $G$ is a compact Lie group, $M$ is a paracompact $C^{1}$-Banach $G$-manifold and $\mathscr{A}$ is some class of spaces having the equivariant $G$-homotopy type 
of $G$-CW-complexes. We shall use that every $G$-CW-complex is a $G$-ANE, i.e. an equivariant absolute neighborhood extensor $[18,12.5]$, and that $M$ is a $G$-ANR. The latter is proved for trivial $G$ in [20, p. 3]. In the general case one can easily obtain it from $[18,8.9]$. When we now prove the continuity of $\mathscr{A}_{\text {-cat }}{ }_{M}$ it is understood that everything happens in the category of $G$-spaces.

Let $X$ be a closed subset of $M, k=\mathscr{A}$-cat ${ }_{M}(X)$ and $\left\{X_{1}, \ldots, X_{k}\right\}$ an $\mathscr{A}$-categorical covering associated to $X \subset M$, i.e. a numerable covering of $X$ such that there are $A_{j} \in \mathscr{A}$ and maps $\alpha_{j}: X_{j} \rightarrow A_{j}, \beta_{j}: A_{j} \rightarrow M$ with $\beta_{j} \alpha_{j}$ homotopic to the inclusion $X_{j} \subset X(j=1, \ldots, k)$. Choose (invariant) open subsets $U_{j}^{\prime \prime}$ of $M$ such that $X_{j}=$ $U_{j}^{\prime \prime} \cap X$. Then $X_{j}$ is closed in the metric space $U_{j}^{\prime \prime}$. Since $A_{j} \in \mathscr{A}$ it has the (equivariant) homotopy type of a $G$-ANE. It follows that up to homotopy $\alpha_{j}$ may be extended to a map $\tilde{\alpha}_{j}: U_{j}^{\prime} \rightarrow A_{j}$ where $U_{j}^{\prime}$ is an open (invariant) neighborhood of $X_{j}$ in $U_{j}^{\prime \prime}$. Consider $\beta_{j} \tilde{\alpha}_{j}: U_{j}^{\prime} \rightarrow M$. Since $M$ is a $G$-ANR there is an open (invariant) neighborhood $U_{j}$ of $X_{j}$ in $U_{j}^{\prime}$ such that $\beta_{j} \tilde{\alpha}_{j} \mid U_{j}$ is (equivariantly) homotopic to the inclusion $U_{j} \subset M$. Now $\left\{U_{1}, \ldots, U_{k}\right\}$ is an $\mathscr{A}$-categorical covering of its union $U$ associated to $U \subset M$, and hence $\mathscr{A}$-cat ${ }_{M}(U)=k$.

\section{REFERENCES}

1. I. Berstein and P. J. Hilton, Category and generalized Hopf invariants, Illinois J. Math. 4 (1960), 437-451.

2. , On suspensions and comultiplications, Topology 2 (1963), 73-82.

3. M. Clapp and D. Puppe, The generalized Lusternik-Schnirelmann category of a product space (in preparation).

4. T. tom Dieck, Partitions of unity in homotopy theory, Compositio Math. 23 (1971), 159-167.

5. T. tom Dieck, K. H. Kamps and D. Puppe, Homotopietheorie, Lecture Notes in Math., vol. 157, Springer-Verlag, Berlin and New York, 1970.

6. E. Fadell, The equivariant Ljusternik-Schnirelmann method for invariant functionals and relative cohomological index theory, Preprint.

7. R. H. Fox, On the Lusternik-Schnirelmann category, Ann. of Math. (2) 42 (1941), 333-370.

8. T. Ganea, A generalization of the homology and homotopy suspension, Comment. Math. Helv. 39 (1965), 295-322.

9. __ Lusternik-Schnirelmann category and strong category, Illinois J. Math. 11 (1967), 417-427.

10. W. J. Gilbert, Some examples for weak category and conilpotency, Illinois J. Math. 12 (1968), 421-432.

11. B. Gray, Homotopy theory. An introduction to algebraic topology, Academic Press, New York, 1975.

12. M. W. Hirsch, Differential topology, Graduate Texts in Math., no. 33, Springer-Verlag, Berlin and New York, 1976.

13. I. M. James, On category in the sense of Lusternik-Schnirelmann, Topology 17 (1978), 331-348.

14. L. Lusternik and L. Schnirelmann, Méthodes topologiques dans les problèmes variationels, Hermann, Paris, 1934.

15. M. Mather, Pull-backs in homotopy theory, Canad. J. Math. 28 (1976), 225-263.

16. L. Montejano, A quick proof of Singhof's $\operatorname{cat}\left(M \times S^{1}\right)=\operatorname{cat}(M)+1$ theorem, Manuscripta Math. 42 (1982), 49-52.

17. Lusternik-Schnirelmann category: A geometric approach, Memoirs of the Topology Semester held at the Banach Center, Warsaw 1984 (to appear).

18. M. Murayama, On G-ANR's and their G-homotopy types, Osaka J. Math. 20 (1983), 479-512.

19. R. S. Palais, Morse theory on Hilbert manifolds, Topology 2 (1963), 299-340.

20. __ Homotopy theory of infinite dimensional manifolds, Topology 5 (1966), 1-16.

21. _ Lusternik-Schnirelmann theory on Banach manifolds, Topology 5 (1966), 115-132.

22. R. S. Palais and S. Smale, A generalized Morse theory, Bull. Amer. Math. Soc. 70 (1964), 165-171. 
23. W. Singhof, Minimal coverings of manifolds with balls, Manuscripta Math. 29 (1979), 385-415.

24. S. Smale, Morse theory and a nonlinear generalization of the Dirichlet problem, Ann. of Math. (2) 80 (1964), 382-396.

25. F. Takens, The Lusternik-Schnirelmann categories of a product space, Compositio Math. 22 (1970), $175-180$.

26. S. Waner, Equivariant homotopy theory and Milnor's theorem, Trans. Amer. Math. Soc. 258 (1980), $351-368$.

27. G. W. Whitehead, The homology suspension, Colloq. Topologie Algébrique (Louvain, 1956), pp. $89-95$.

Instituto de Matemáticas, U.N.A.M., Ciudad Universitaria, 04510 Mexico, D. F., Mexico

Mathematisches Institut der Universität Im Neuenheimer Feld 288, D-6900 Heidelberg, FEDERAL RepUblic OF GERMANY 